\title{
Real-Time Motor Cortex Mapping for the Safe Resection of Glioma: An Intraoperative Resting-State fMRI Study
}

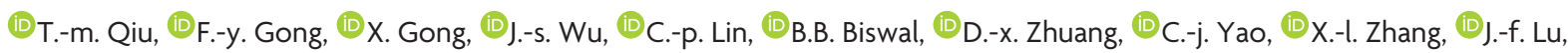 \\ (1)F.-p. Zhu, 1 Y. Mao, and $\mathbb{D}_{\text {L.f.f. Zhou }}$
}

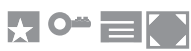

\begin{abstract}
BACKGROUND AND PURPOSE: Resting-state functional MR imaging has been used for motor mapping in presurgical planning but never used intraoperatively. This study aimed to investigate the feasibility of applying intraoperative resting-state functional MR imaging for the safe resection of gliomas using real-time motor cortex mapping during an operation.
\end{abstract}

MATERIALS AND METHODS: Using interventional MR imaging, we conducted preoperative and intraoperative resting-state intrinsic functional connectivity analyses of the motor cortex in 30 patients with brain tumors. Factors that may influence intraoperative imaging quality, including anesthesia type (general or awake anesthesia) and tumor cavity (filled with normal saline or not), were studied to investigate image quality. Additionally, direct cortical stimulation was used to validate the accuracy of intraoperative resting-state fMRI in mapping the motor cortex.

RESULTS: Preoperative and intraoperative resting-state fMRI scans were acquired for all patients. Fourteen patients who successfully completed both sufficient intraoperative resting-state fMRI and direct cortical stimulation were used for further analysis of sensitivity and specificity. Compared with those subjected to direct cortical stimulation, the sensitivity and specificity of intraoperative resting-state fMRI in localizing the motor area were $61.7 \%$ and $93.7 \%$, respectively. The image quality of intraoperative resting-state fMRI was better when the tumor cavity was filled with normal saline $(P=.049)$. However, no significant difference between the anesthesia types was observed $(P=.102)$.

CONCLUSIONS: This study demonstrates the feasibility of using intraoperative resting-state fMRI for real-time localization of functional areas during a neurologic operation. The findings suggest that using intraoperative resting-state fMRI can avoid the risk of intraoperative seizures due to direct cortical stimulation and may provide neurosurgeons with valuable information to facilitate the safe resection of gliomas.

ABBREVIATIONS: BOLD = blood oxygen level-dependent; $D C S$ = direct cortical stimulation; $\mathrm{MRI}=$ intraoperative MR imaging; iR-fMRI = intraoperative resting-state fMRI; $\mathrm{PR}$-fMRI = preoperative resting-state fMRI; R-fMRI = resting-state fMRI

M apping the motor cortex before and during tumor resection is of great importance to minimize the risks of postoperative neurologic sequelae. Direct cortical stimulation (DCS) is an invasive procedure to locate the function of specific brain regions. Due to its simplicity, DCS is considered the clinical criterion standard for mapping brain function and has demonstrated efficacy

Received February 3, 2017; accepted after revision June 25.

From the Department of Neurological Surgery (T.-m.Q., F.-y.G., X.G., J.-s.W., D.-x.Z., C.-j.Y., X.-I.Z., J.-f.L., F.-p.Z., Y.M., L.-f.Z.), Huashan Hospital, Shanghai Medical College, Fudan University, Shanghai, China; Center for Computational Systems Biology (C.-p.L.), Fudan University, Shanghai, China; and Department of Biomedical Engineering (B.B.B.), New Jersey Institute of Technology, Newark, New Jersey.

Tian-ming Qiu and Fang-yuan Gong contributed equally to this article.

This work was funded by the National Natural Science Foundation of China (grant No. 81401546, 81671308, 81672476), Natural Science Foundation and Major Basic Research Program of Shanghai (grant No. 16JC1420100), and National Key Technology R\&D Program of China (grant No, 2014BAI04B05). in optimizing glioma resection. ${ }^{1,2}$ However, as an invasive approach, DCS requires a surgical team with rich experience. DCS also has the risk of after discharges, which can induce seizures and result in the inaccurate localization of cortical areas. ${ }^{3}$

Blood oxygen level-dependent (BOLD) functional MR imaging, a task-based brain functional mapping method, has been 
well-established for localizing the brain functional area for presurgical planning. BOLD fMRI is of great importance in helping decrease morbidity due to a neurologic operation ${ }^{4}$ and has been integrated into neuronavigation systems to localize the motor area during an operation. ${ }^{5}$ Nonetheless, brain shifting during an operation might reduce the accuracy of preoperative imaging and affect the clinical consequences. ${ }^{6}$

The emergence of intraoperative MR imaging (iMRI) has ushered in a new era in brain tumor neurosurgery. ${ }^{7}$ Real-time structural imaging and diffusion tensor imaging have provided neurosurgeons with valuable information regarding whether and where tumor residues persist after resection and even the relationship between the tumor/tumor cavity and peritumoral tracts. ${ }^{8,9}$ The use of an iMRI navigation system can reliably compensate for the effects of brain shifting. ${ }^{6}$ We have recently reported intraoperative motor mapping with $\mathrm{PMRI}$ for the first time; in this procedure, awake intraoperative fMRI was used to localize the sensorimotor areas during awake craniotomy. ${ }^{10}$ Cooperation of the patient under specific tasks and complicated surgical procedures is essential for successful mapping. Therefore, task-induced brain mapping cannot be achieved with the patient under general anesthesia.

Resting-state fMRI (R-fMRI) has recently been used to identify the motor cortex without a task stimulus. ${ }^{11}$ This technique has been used in preoperative motor mapping in patients with brain tumors. ${ }^{12-14}$ Our previous study demonstrated the accuracy of preoperative R-fMRI ( $\mathrm{pR}-\mathrm{fMRI}$ ) for motor area localization by DCS before tumor resection. ${ }^{13}$ However, the study was based on $\mathrm{pR}-\mathrm{fMRI}$ and lacked real-time information regarding the relationship between the tumor cavity/residual and the motor cortex, which might have affected the conclusion of whether further resection is safe.

To our knowledge, functional connectivity based on intraoperative resting-state fMRI (iR-fMRI) has not been applied to real-time motor cortex mapping during an operation. The purpose of this study was to investigate the feasibility and validity of applying iR-fMRI to neurosurgical mapping. The sensitivity and specificity of iR-fMRI in mapping the motor cortex were assessed and compared with those of DCS. The optimum iR-fMRI protocol for better intraoperative imaging quality was also studied.

\section{MATERIALS AND METHODS \\ General Information}

The study examined 30 patients with cerebral tumors involving the motor cortical areas. The patients were recruited and enrolled in this study by the Neurologic Surgery Department at Huashan Hospital, Shanghai. The study was reviewed and approved by the institutional review board of Huashan Hospital.

The patients included 19 men and 11 women 19-70 years of age. The inclusion criteria were as follows: 1) patients with a single lesion and first operation, 2) tumor lesions in the frontal or parietal lobe near (or involving) the precentral gyrus, and 3) no contraindications for MR imaging or intraoperative electrophysiologic monitoring. The exclusion criteria were as follows: 1) recurrent tumors, 2) contraindications to MR imaging or intraoperative electrophysiologic monitoring, 3) preoperative muscle strength at $0^{\circ}$, and 4) an inability to comply with safety screening requirements during intraoperative MR imaging screening. Each patient's muscle strength was recorded before the operation and within 3 months after it. Muscle function was evaluated and assigned a grade according to the scale of $0-5$.

\section{MR Imaging Data Acquisition}

Preoperative and intraoperative images were acquired with a 3T interventional MR imaging scanner (Trio; Siemens, Erlangen, Germany) at Huashan Hospital. Preoperative images were obtained 1-2 days before the date of the operation. Enhanced T1weighted images were acquired to differentiate patients with high- or low-grade gliomas with the following imaging parameters: $\mathrm{TR}=1900 \mathrm{~ms}$; $\mathrm{TE}=2.93 \mathrm{~ms}$; flip angle $=90^{\circ}$; section number $=176$; section thickness $=1 \mathrm{~mm}$; and $\mathrm{FOV}=250 \times$ $219 \mathrm{~mm}$. The T2-weighted fluid-attenuated inversion recovery images were acquired with multishot $\mathrm{TSE}$ sequences with $\mathrm{TR}=$ $9000 \mathrm{~ms}, \mathrm{TE}=99 \mathrm{~ms}$, TI $=2500 \mathrm{~ms}$, flip angle $=150^{\circ}$, section number $=66$, section thickness $=2 \mathrm{~mm}$, and FOV $=240 \times$ $214 \mathrm{~mm}$.

The R-fMRI scans were obtained with a T2-weighted gradientecho-planar imaging sequence with TR/TE $=2000 / 35 \mathrm{~ms}$, flip angle $=90^{\circ}$, matrix size $=64 \times 64, \mathrm{FOV}=240 \times 240 / 210 \times 210$ $\mathrm{mm}$ (preoperative/intraoperative), 33/25 (preoperative/intraoperative) interleaved axial sections oriented along the anterior/posterior commissure line without intersection gap, and 240 continuous image volumes. The participants were instructed to relax, keep their eyes closed without falling asleep, and not think of anything.

\section{Intraoperative MR Imaging Environment}

All the operations were performed in a 3T iMRI-integrated operating room (IMRIS, Winnipeg, Manitoba, Canada) at a constant temperature of $20^{\circ}-21^{\circ} \mathrm{C}$. Because the intraoperative imaging process has been detailed in our previous article, it is only briefly described here. ${ }^{9}$ The patient's head was fixed with an MR imaging-safe head holder before the craniotomy. DCS was used to localize the motor cortex after opening the dura. Sodium valproate was used to prevent seizures. After tumor resection and wound draping, the scanner was moved into the operation room. In the cases that required further resection, the navigational images were updated with iMRI datasets.

To optimize the image quality, we studied factors that may influence intraoperative imaging quality, including anesthesia type and whether the tumor cavity was filled with normal saline during scanning. Twelve of the 30 patients underwent general anesthesia, and the other 18 patients underwent awake anesthesia. General anesthesia was administered intravenously using remifentanil as an analgesic and propofol as a sedative via endotracheal intubation. The 18 patients who underwent awake anesthesia were administered midazolam for sedation combined with local nerve block anesthesia with remifentanil as an analgesic and propofol as a sedative. For the tumor cavity process, 12 patients' tumor cavities were filled with normal saline and covered with wet gauze. The tumor cavities of the other 18 patients were only covered with wet gauze without filling. 


\section{Intraoperative DCS}

DCS was performed by using a Multifunctional Neurologic Workstation (Epoch XP; Axon Systems, Hauppauge, New York) to locate the motor cortex in all 30 patients before tumor resection. The electric current was increased gradually from 2 to $6 \mathrm{~mA}$ in 1-mA increments. After discharge, the current was decreased by $0.5-1 \mathrm{~mA}$. During the stimulation process, the compound muscle action potentials were monitored, including those from the abductor pollicis brevis, brachioradialis, triceps, biceps, tibialis anterior, gastrocnemius, and orbicularis oris muscles.

When the compound muscle action potentials or passive movements of the target muscle occurred, the representative area in the cortex corresponding to the movement was identified and noted with a sterile tag. A $10 \times 10 \mathrm{~mm}$ area on the exposed cortical surface was considered 1 unit of stimulation. The corresponding motor sites in structural images were then recorded by a navigation system (TRIA i7; Medtronic Navigation, Minneapolis, Minnesota).

\section{R-fMRI Data Processing}

The fMRI datasets were preprocessed and analyzed by Data Processing Assistant for Resting-State fMRI, Advanced Edition (Beijing Normal University, Beijing, China), ${ }^{15}$ Resting-State fMRI Data Analysis Toolkit (Beijing Normal University), ${ }^{16}$ and SPM8 (http://www.fil.ion.ucl.ac.uk/spm/software/spm12). The results were visualized with XjView 8 (http://www.alivelearn.net/xjview).

pR-fMRI were preprocessed by removal of the first 10 time points. This process was followed by section-timing correction, realignment, registration, smoothing, and detrending to reduce the impact of the magnetic field environment or patient instability on the images. A temporal bandpass filter $(0.01$ $\mathrm{Hz}<\mathrm{f}<0.08 \mathrm{~Hz}$ ) was then applied to remove low-frequency drifts and high-frequency noise. The spurious BOLD variances unlikely to reflect neuronal activity were regressed. These variances included signals from the CSF, white matter, and whole brain, as well as the 6 parameters obtained by rigid-body head motion correction. ${ }^{17}$

A 3-mm-diameter seed mask was placed in the hand-knob area of the healthy (nontumor) side, ${ }^{18}$ which was identified from high-resolution structural MR imaging and confirmed as a landmark of the precentral gyrus of the brain under normal or disease conditions. ${ }^{19}$ Functional connectivity was computed between the selected seed masks, from which time-series signals were averaged and correlated with every voxel in the brain. The threshold and spatial extent of activation corresponding to this threshold were determined by a neuroradiologist. This process was performed by maximizing the functional neuronal activity while minimizing nonspecific noise patterns. ${ }^{12}$

iR-fMRI processing was identical to the $\mathrm{pR}$-fMRI processing. However, because the patient's head was fixed in the holder, motion-induced signal changes were minimal. We required approximately 15 minutes on average to process the data immediately after scanning. During this 15-minute period, we moved the scanner out, redraped the patient, and updated the intraoperative navigation images.

\section{Statistical Analyses}

Data analysis was performed by using PASW18.0 statistical software (IBM, Armonk, New York). DCS-positive sites were labeled on iMRI structural images for motor-related tags and were used for comparison. We regarded the following DCS results as "unsatisfactory": 1) Seizure occurred, and intraoperative mapping was stopped; 2) the intracranial pressure was high after opening the dura; therefore, we only stimulated the cortex corresponding to the potential motor area and did not stimulate all the sites within the exposed cortical surface; and 3) both DCS and R-fMRI had negative results within the exposed cortical surface.

To optimize the imaging quality, we grouped and compared patients with different anesthesia types and tumor cavity fillings We regarded the following R-fMRI results as showing "unsatisfactory" image quality: 1) A highly correlated functional connectivity map was found in the ventricle, tumor/tumor cavity, or scalp; and 2) obvious irrational motor functional connectivity was found. The Fisher exact test was used to assess differences in image quality by scanning situation. A value of .05 indicated significance.

We chose the cases with satisfactory fMRI and DCS results to calculate the sensitivity and specificity of iR-fMRI. A match was identified when a significant overlap between the $10 \times 10 \mathrm{~mm}$ scope around the DCS-positive sites and functional areas was observed on the fMRI. For each patient, a $10 \times 10 \mathrm{~mm}$ area on the exposed cortical surface was considered 1 unit for analysis. For each case, with the DCS-positive sites as references, we calculated the number of true-positive, true-negative, false-positive, and false-negative tags. Each cortical site on the DCS map was considered independently. A method for analyzing clustered binary data was used to calculate the sensitivity and specificity of iR-fMRI. This method was developed by Bizzi et $\mathrm{al}^{20}$ and Roux et al. ${ }^{21}$

\section{RESULTS}

All 30 patients underwent preoperative and intraoperative MR imaging and DCS (On-line Table 1). Overall, 27 (of 30) patients had satisfactory pR-fMRI motor mapping, and 21 (of 30) patients had satisfactory iR-fMRI motor mapping. The 9 patients with unsatisfactory iR-fMRI included 3 patients with a highly correlated functional connectivity map found in the tumor/tumor cavity or scalp (cases 4,11, and 17), 5 patients with obvious irrational motor functional connectivity (cases 10, 13, 15, 16, and 23), and 1 patient with both of the above situations (case 28). Motor mapping with DCS was achieved in 18 cases without high intracranial pressure after craniotomy and no seizures during mapping. Fourteen patients who successfully completed both sufficient iR-fMRI and DCS were used for further analysis to determine the sensitivity and specificity. We listed all the DCS and iR-fMRI results for each site on the exposed cortical surface for each case. On the basis of the clustered binary data above, we constructed a 4 -fold table (On-line Table 2 ) with the sum from true-positives, true-negatives, false-positives, and falsenegatives (Table) and then calculated the sensitivity and specificity. The sensitivity and specificity of iR-fMRI were $61.7 \%$ (95\% CI, 49.02\%-72.91\%) and 93.7\% (95\% CI, 88.74\%-96.53\%), respectively (Table and On-line Table 1). 
Comparison of the results from iR-fMRI and DCS for 14 of the 30 patients

\begin{tabular}{lcrcc}
\hline & \multicolumn{4}{c}{ Intraoperative R-fMRI } \\
\cline { 2 - 5 } No. & TP & TN & FP & FN \\
\hline 2 & 9 & 7 & 0 & 0 \\
5 & 3 & 8 & 3 & 0 \\
8 & 1 & 22 & 1 & 0 \\
12 & 1 & 8 & 1 & 3 \\
18 & 0 & 19 & 0 & 1 \\
19 & 2 & 19 & 0 & 3 \\
20 & 4 & 8 & 0 & 3 \\
21 & 1 & 14 & 2 & 0 \\
22 & 4 & 3 & 1 & 0 \\
25 & 2 & 4 & 0 & 3 \\
26 & 3 & 10 & 2 & 1 \\
27 & 3 & 12 & 0 & 1 \\
29 & 2 & 8 & 0 & 4 \\
30 & 2 & 6 & 0 & 4 \\
\hline
\end{tabular}

Note:-TP indicates true-positive; TN, true-negative; FP, false-positive; FN, falsenegative.

To identify differences in image quality by scanning situation, we filled the tumor cavities of 12 patients with normal saline and compared the cavities with those of the other 18 patients who did not undergo cavity filling. Our results showed that a tumor cavity filled with normal saline could improve the imaging quality $(P=$ .049). In addition, 12 and 18 patients were selected to undergo general and awake anesthesia, respectively. No significant correlation between anesthesia type and imaging quality was found $(P=.102)$. Thus, the imaging quality of iR-fMRI was superior when the tumor cavity was filled with normal saline but was not significantly correlated with anesthesia type. We have provided 4 illustrative cases as on-line images, including 2 cases of iR-fMRI without saline in the tumor cavity (On-line Figs 1 and 2), and 2 cases of iR-fMRI with saline (On-line Figs 3 and 4). These raw data showed how image quality changed because of saline in the intraoperative cavity.

Six patients experienced motor deficits (muscle strength decreased) immediately after the operation, with 4 of the 6 recovering to their preoperative condition within 3 months.

\section{Case 1}

A 41-year-old woman (case 22 in On-line Table 1) had recurrent seizures for $>2$ months with normal muscle strength before the operation. MR imaging showed that the lesion was in the right frontal lobe, while the pR-fMRI scan revealed that the motor cortex was located posteriorly (Fig $1 A$ ). The patient underwent awake craniotomy and DCS to locate 2 positive sites in the hand area and 2 in the mouth area (Fig $1 B$ ). The positive sites were recorded on the MR images and overlaid with the motor cortex displayed on the pR-fMRI. The results agreed with the pR-fMRI findings (Fig 1A). After tumor resection, the navigation was updated by the intraoperative image, while the positive sites located by DCS were recorded by navigation on the iMRI and overlaid with the motor cortex displayed on the iR-fMRI. The results demonstrated that the positive sites still agreed with the iR-fMRI results (Fig 1C). After the operation, the patient had mild loss of muscle strength but recovered to normal after 1 week.

\section{Case 2}

A 25-year-old man (case 5 in On-line Table 1) had general seizures for 2 months with normal muscle strength before the operation. The preoperative MR imaging showed that the tumor was in the left frontal lobe, while the R-fMRI revealed that the motor cortex was posterior to the lesion (Fig $2 A$ ). The DCS identified 2 positive sites in the hand area, 1 in the foot area, and 2 in the language area corresponding to speech fluency (Fig 2B). After tumor resection, iMRI indicated a small amount of tumor residue close to the deep motor area behind the tumor cavity (Fig 2D), while the iR-fMRI showed some distance between the motor cortex and tumor residue (Fig 2C). Accordingly, another resection was performed, and the subsequent iMRI scan demonstrated that the tumor was completely removed (Fig $2 F$ ). The iR-fMRI scan showed that the motor cortex was well-protected (Fig 2E). With iR-fMRI guidance, further resection was safely performed. The patient had transient slow right-limb movement start-up that gradually improved to normal.

\section{DISCUSSION}

High-field iMRI is an innovative technique to aid the functional guidance of neurosurgery. It is a challenge for patients to perform tasks during the operation. R-fMRI provides a window for identifying the motor cortex without the patient's cooperation. The combination of iMRI and R-fMRI (ie, iR-fMRI) was applied in this study. Intraoperative mapping of regional brain functions with iR-MR imaging was validated by DCS. This new intraoperative technique is noninvasive compared with DCS. Our results indicated that iR-fMRI might be a promising tool for real-time motor cortex mapping during a glioma operation.

Accumulating evidence has suggested that more extensive surgical resection is associated with a longer life expectancy for patients with gliomas. ${ }^{22,23}$ In addition, surgical morbidities may also affect survival time. Preoperative and intraoperative care should be taken to avoid surgical sequelae in the management of gliomas. ${ }^{24,25}$ Accurate real-time positioning of intraoperative brain function is among the most important issues for neurosurgeons. In recent studies, R-fMRI was found to be well-correlated with the pre- and postoperative clinical condition of patients. ${ }^{26}$ DCS serves as a reliable method to locate brain function areas, and it has been popularized. However, repeat stimulations may greatly increase the incidence of seizure, reduce surgical safety, and directly affect DCS accuracy. ${ }^{3,27}$ Notably, DCS cannot really be applied after partial tumor removal. Noninvasive iR-fMRI, which was verified by DCS in the current research, may provide neurosurgeons with important information conducive to safe tumor resection, especially for patients who cannot cooperate with DCS and task fMRI. Our results indicated that the fusion of functional and structural findings could clearly reveal the spatial location of the tumor residue and motor cortex, information valuable to neurosurgeons in assessing whether further resection is feasible and safe.

Our results demonstrated that $70 \%$ of iR-fMRI quality was satisfactory for neurosurgery reference. However, $30 \%$ of the iRfMRI data did not meet the criteria for acceptance. Our results indicated that the imaging quality was not significantly affected by the anesthesia type. The lack of effect from anesthesia on R-fMRI 

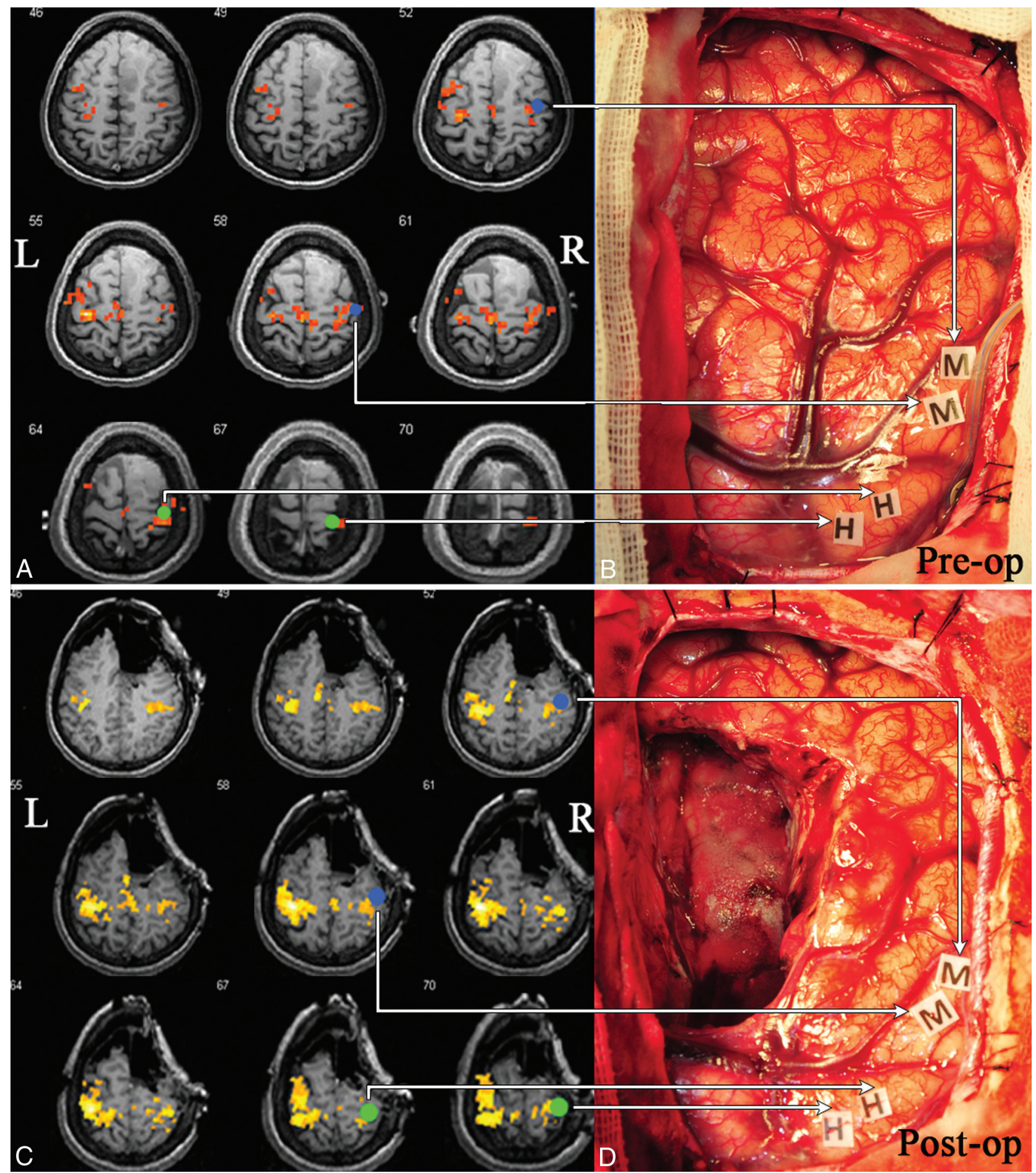

FIG 1. Validation of $\mathrm{RR}-\mathrm{fMRI}$ with DCS. A, The $\mathrm{PR}-\mathrm{fMRI}$ scan shows the motor cortex located posterior to the tumor. $B$, DCS locates positive sites in the hand area $(\mathrm{H})$ and positive sites in the mouth area (M). The green and blue dots in $A$ represent the positive sites in the hand and mouth areas, respectively, located by intraoperative DCS. C, iR-fMRI reveals that the motor cortex is behind the tumor cavity. D, Intact motor cortex after tumor resection. The green and blue dots represent the positive sites in the hand and mouth areas, respectively, located by intraoperative DCS. Pre-op indicates preoperative; Post-op, postoperative; L, left; R, right.

signal has also been found in other studies. ${ }^{26,28}$ The image quality, however, could be improved by filling the tumor cavity with normal saline. This improvement might result from the creation of a homogeneous imaging environment, minimizing the susceptibility artifacts during iR-fMRI acquisition. However, not all patients who underwent normal saline filling had satisfactory iR-fMRI quality. Whether normal saline is the most ideal substance for tumor cavity filling remains questionable.

The iR-fMRI scan was useful for guidance of brain tumor operations but still had several limitations. The BOLD signal, which is observed using the blood oxygen level to reflect brain activation, cannot directly show the electrical activities of neurons, a finding 

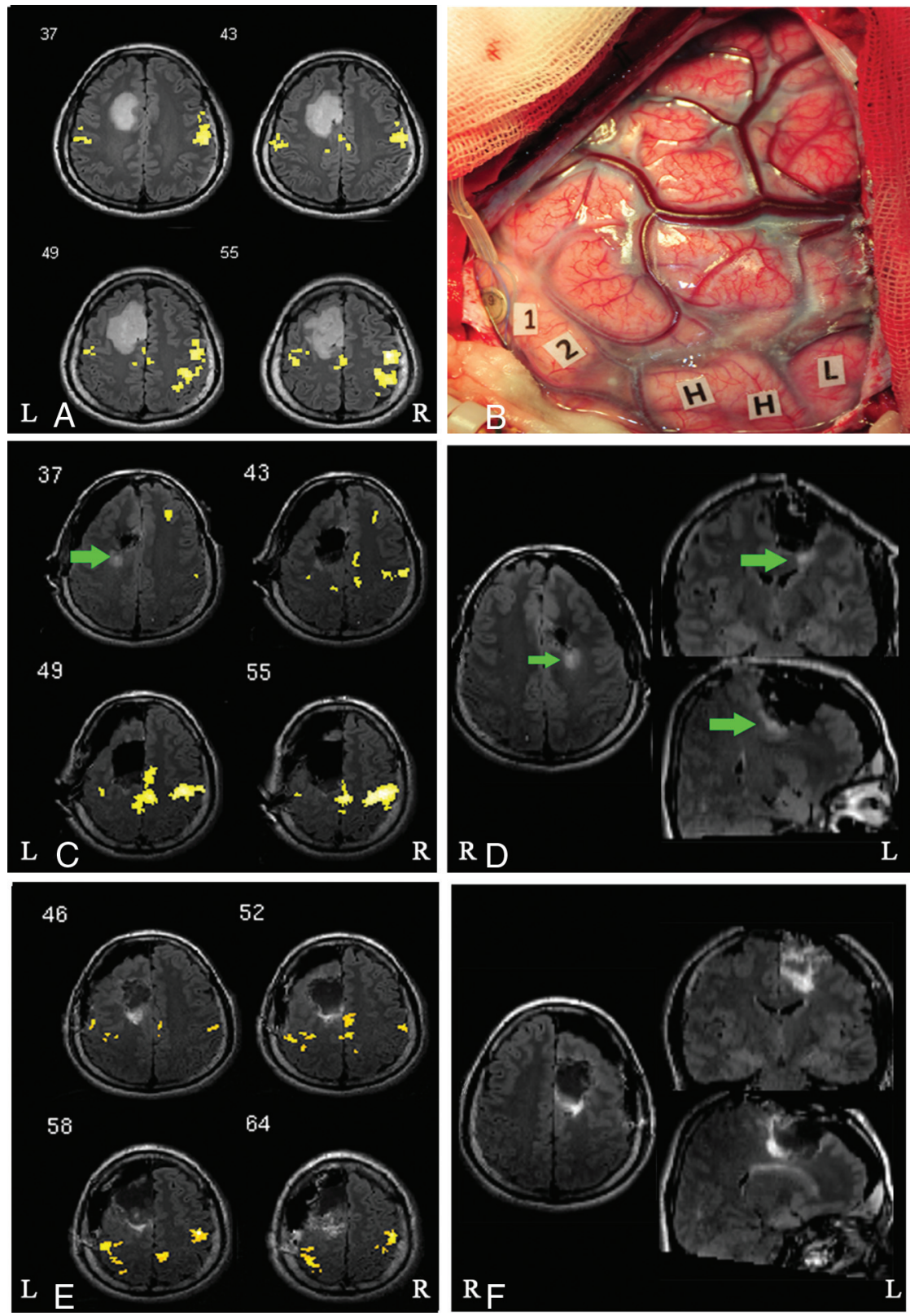

FIG 2. Procedure for iR-fMRI- and DCS-assisted tumor resection. $A$, pR-fMRI scan reveals that the motor cortex is located behind the left frontal lesion. B, Direct cortical stimulation locates 2 positive sites in the hand area $(\mathrm{H}), 1$ positive site in the foot area (L), and 2 positive sites in the language area (1 and 2). C, The iR-fMRI scan displays the motor cortex behind the tumor cavity at a distance from the tumor residue (green arrow). D, Intraoperative MR image suggests a small amount of tumor residue closer to the deep motor area behind the tumor cavity (green arrow). $E$, The final iR-fMRI scan shows that the motor cortex located behind the tumor cavity is wellprotected. $F$, The final MR imaging scan reveals complete tumor resection. $L$ indicates left; R, right. in this study. In recent studies, breathholding fMRI was used to calibrate resting-state fMRI or task-based fMRI to reduce tumor-related neurovascular factors. We have been attempting to use this method to try to obtain better results. Additionally, this is a relatively small sample, which may be responsible for the trend level. We are attempting to collect additional cases and obtain significant results. Further studies are essential to solve these problems to ensure that iR-fMRI becomes a safe and effective tool for clinical applications. We are currently exploring other analysis methods, including amplitude of low frequency fluctuation, regional homogeneity, and independent component analysis, ${ }^{12,30}$ to further optimize the analysis.

\section{CONCLUSIONS}

This study investigated the iR-fMRI technique for the intraoperative localization of the motor cortex. Although this research is in its infancy, we demonstrated the feasibility of applying iRfMRI for real-time functional localization. The areas located by iR-fMRI provided the motor regions that overlapped with the corresponding areas identified by DCS. Therefore, iR-fMRI provides an alternative opportunity for real-time mapping of motor areas and increases the safety of the resection of intra-axial brain tumors.

\section{ACKNOWLEDGMENTS}

The authors thank Jian-bing Shi and Zhong Yang for assembling the image data base, Wei-jun Tang for checking the data processing, Gen Xu for providing the direct cortical stimulation, and Yan-yan Song and Chun-xiao Wu for performing the statistical analyses. that is different from that in DCS. However, BOLD has irreplaceable significance in localizing functional areas when DCS does not work or a seizure occurs. The results of the seed-based method could be assessed in real-time. Nonetheless, we also found that a prominently decreased ipsilesional BOLD signal was present compared with that in the normal side. We supposed that this finding may be attributed to tumor-induced neurovascular uncoupling. ${ }^{29}$ Abnormal vasculature due to tumors causes significant hemodynamic responses in the cerebrovascular reactivity that affect the BOLD signal, resulting in false-negative results, which may explain why the overall sensitivity was not very high

\section{REFERENCES}

1. Matz PG, Cobbs C, Berger MS. Intraoperative cortical mapping as a guide to the surgical resection of gliomas. J Neurooncol 1999;42: 233-45 CrossRef Medline

2. Yetkin FZ, Mueller WM, Morris GL, et al. Functional MR activation correlated with intraoperative cortical mapping. AJNR Am J Neuroradiol 1997;18:1311-15 Medline

3. Trebuchon A, Guye M, Tcherniack V, et al. Interest of EEG recording during direct electrical stimulation for brain mapping function in surgery [in French]. Ann Fr Anesth Reanim 2012;31:e87-90 CrossRef Medline

4. Vlieger EJ, Majoie CB, Leenstra S, et al. Functional magnetic resoAJNR Am J Neuroradiol 38:2146-52 Nov 2017 www.ajnr.org 
nance imaging for neurosurgical planning in neurooncology. Eur Radiol 2004;14:1143-53 CrossRef Medline

5. Krishnan R, Raabe A, Hattingen E, et al. Functional magnetic resonance imaging-integrated neuronavigation: correlation between lesion-to-motor cortex distance and outcome. Neurosurgery 2004; 55:904-14; discusssion 914-15 Medline

6. Nimsky C, Ganslandt O, Cerny S, et al. Quantification of, visualization of, and compensation for brain shift using intraoperative magnetic resonance imaging. Neurosurgery 2000;47:1070-79; discussion 1079-80 CrossRef Medline

7. Black PM, Moriarty T, Alexander E 3rd, et al. Development and implementation of intraoperative magnetic resonance imaging and its neurosurgical applications. Neurosurgery 1997;41:831-42; discussion 842-45 CrossRef Medline

8. Nimsky C, Ganslandt O, Hastreiter P, et al. Preoperative and intraoperative diffusion tensor imaging-based fiber tracking in glioma surgery. Neurosurgery 2007;61:178-85; discussion 186 Medline

9. Qiu TM, Yao CJ, Wu JS, et al. Clinical experience of 3T intraoperative magnetic resonance imaging integrated neurosurgical suite in Shanghai Huashan Hospital. Chin Med J (Engl) 2012;125:4328-33 Medline

10. Lu JF, Zhang H, Wu JS, et al. “Awake” intraoperative functional MRI (ai-fMRI) for mapping the eloquent cortex: is it possible in awake craniotomy? Neuroimage Clin 2012;2:132-42 CrossRef Medline

11. Biswal B, Yetkin FZ, Haughton VM, et al. Functional connectivity in the motor cortex of resting human brain using echo-planar MRI. Magn Reson Med 1995;34:537-41 CrossRef Medline

12. Shimony JS, Zhang D, Johnston JM, et al. Resting-state spontaneous fluctuations in brain activity: a new paradigm for presurgical planning using fMRI. Acad Radiol 2009;16:578 - 83 CrossRef Medline

13. Qiu TM, Yan CG, Tang WJ, et al. Localizing hand motor area using resting-state fMRI: validated with direct cortical stimulation. Acta Neurochir (Wien) 2014;156:2295-302 CrossRef Medline

14. Schneider FC, Pailler M, Faillenot I, et al. Presurgical assessment of the sensorimotor cortex using resting-state fMRI. AJNR Am J Neuroradiol 2016;37:101-07 CrossRef Medline

15. Chao-Gan Y, Yu-Feng Z. DPARSF: A MATLAB Toolbox for "Pipeline" data analysis of resting-state fMRI. Front Syst Neurosci 2010; 4:13 CrossRef Medline

16. Song XW, Dong ZY, Long XY, et al. REST: a toolkit for resting-state functional magnetic resonance imaging data processing. PLoS One 2011;6:e25031 CrossRef Medline

17. Fox MD, Raichle ME. Spontaneous fluctuations in brain activity observed with functional magnetic resonance imaging. Nat Rev Neurosci 2007;8:700-11 CrossRef Medline
18. Boroojerdi B, Foltys H, Krings T, et al. Localization of the motor hand area using transcranial magnetic stimulation and functional magnetic resonance imaging. Clin Neurophysiol 1999;110:699-704 CrossRef Medline

19. Yousry TA, Schmid UD, Alkadhi H, et al. Localization of the motor hand area to a knob on the precentral gyrus: a new landmark. Brain 1997;120(pt 1):141-57 CrossRef Medline

20. Bizzi A, Blasi V, Falini A, et al. Presurgical functional MR imaging of language and motor functions: validation with intraoperative electrocortical mapping. Radiology 2008;248:579-89 CrossRef Medline

21. Roux FE, Boulanouar K, Lotterie JA, et al. Language functional magnetic resonance imaging in preoperative assessment of language areas: correlation with direct cortical stimulation. Neurosurgery 2003;52:1335-45; discussion 1345-47 CrossRef Medline

22. Smith JS, Chang EF, Lamborn KR, et al. Role of extent of resection in the long-term outcome of low-grade hemispheric gliomas. J Clin Oncol 2008;26:1338-45 CrossRef Medline

23. Sanai N, Berger MS. Glioma extent of resection and its impact on patient outcome. Neurosurgery 2008;62:753-64; discussion 764-66 CrossRef Medline

24. De Witt Hamer PC, Robles SG, Zwinderman AH, et al. Impact of intraoperative stimulation brain mapping on glioma surgery outcome: a meta-analysis. J Clin Oncol 2012;30:2559-65 CrossRef Medline

25. Sanai N, Mirzadeh Z, Berger MS. Functional outcome after language mapping for glioma resection. N Engl J Med 2008;358:18-27 CrossRef Medline

26. Roder C, Charyasz-Leks E, Breitkopf M, et al. Resting-state functional MRI in an intraoperative MRI setting: proof of feasibility and correlation to clinical outcome of patients. J Neurosurg 2016;125: 401-09 CrossRef Medline

27. Blume WT, Jones DC, Pathak P. Properties of after-discharges from cortical electrical stimulation in focal epilepsies. Clin Neurophysiol 2004;115:982-89 CrossRef Medline

28. Bisdas S, Charyasz-Leks E, Roder C, et al. Evidence of resting-state activity in propofol-anesthetized patients with intracranial tumors. Acad Radiol 2016;23:192-99 CrossRef Medline

29. Agarwal S, Sair HI, Yahyavi-Firouz-Abadi N, et al. Neurovascular uncoupling in resting state fMRI demonstrated in patients with primary brain gliomas. J Magn Reson Imaging 2016;43:620-66 CrossRef Medline

30. Kokkonen SM, Nikkinen J, Remes J, et al. Preoperative localization of the sensorimotor area using independent component analysis of resting-state fMRI. Magn Reson Imaging 2009;27:733-40 CrossRef Medline 